\title{
Accessibility for mental healthcare
}

Keywords: mental healthcare facilities, design, accessibility, universal design.

\begin{abstract}
Purpose: Patients' movement in mental health facilities is frequently compromised for reasons quite apart from real physical incompetence. The paper finds that accessibility within mental healthcare facilities is a more complex an issue than universal accessibility standards generically allow for.
\end{abstract}

Design/methodology/approach: Several community mental healthcare units (in both Great Britain and France) are reviewed and analysed while they are occupied and running. The focus of the study is on restrictions of movement and the use of universal accessibility devices. The data is part of a broader exploratory study of facilities for mental healthcare, which used empirical, comparative and user inclusive methods.

Findings: Mental health facilities are rarely designed for the model of care and staffing regimes which they will house. This discordance between the physical and organizational milieu inevitably compromises accessibility, even though patients tend to be physically able. Outdoor access, vertical circulation and the accessibility of bathrooms are particularly affected.

Research limitations and implications: Models of care, management and staffing requirements, therapeutic needs of patients and interpersonal relationships should be considered for accessibility during planning, in addition to traditional accessibility devices and design. Furthermore more research is needed to address the ways that accessibility devices need to be altered to comply with the psychosocial elements.

Originality/value: This paper readdresses the traditional view of accessibility, suggesting the paradigm needs to be better developed and nuanced for mental healthcare facilities.

Acknowledgements: This work was supervised by Professor Julienne Hanson at the Bartlett School of Graduate Studies, UCL and sponsored by the Alexander S. Onassis Public Benefit Foundation. 


\section{Introduction}

The accessibility of an environment is the sum of conditions that make an environment available and usable to users. Architecturally, accessibility is associated with building codes and statutes, devices and design standards that extend the usability of a space in consideration of people with impaired mobility. But while mental health patients are generally physically able, they are challenged in a host ways that other users aren't. In mental health facilities built to current paradigms, patient mobility is often severely curtailed, although usually not because of obvious physical disabilities.

Most of the available literature on mobility and mental health concerns organic conditions such as dementia, brain-related syndromes, mental disabilities and psychogeriatrics, but the behaviour of mentally ill patients is usually more unpredictable than the behaviour of the elderly, physically impaired and those with organic brain conditions. And while the available literature may still be relevant, particularly when comorbidities may mean a patient is both disabled and mentally ill, such speculations are beyond the scope of this article. As such it is better to consult the available research directly. For dementia please see Bell (1992) and more specifically for Alzheimer's disease see Zeisel (2006, 2010). For psychogeriatrics see Sloan Devlin (1992) and Dawson (1993). For way-finding considerations see Mahnke (1996). For children with developmental disabilities see Saphiro et al $(1997,2001)$ and for highly sensitive people see Aron (1998).There is also literature on barrier-free housing and lifetime housing, a concept in which various forms of disability are taken into consideration during the design stage for domestic buildings - especially for the elderly (Kelly, 1993, Pollack, 1997). Once again, this subject is beyond the concerns of this article.

The mentally ill tend to be more able than people with physical disability problems as, to a great extent, they are peripatetic. In some cases (for instance in about $6.9 \%$ of schizophrenia patients (Van der Heijden et all, 2005), will still encounter mobility issues due to their pathology, but in these instances, universal accessibility devices are unlikely to be of assistance. Catatonic conditions (a symptom of catatonic 
schizophrenia, catatonic bipolar disorder, Neuroleptic Malignant Syndrome and occasionally in other mental conditions) are defined as "a marked decrease in reactivity to the environment, sometimes reaching an extreme degree of complete unawareness (catatonic stupor), maintaining a rigid posture and resisting efforts to be moved (catatonic rigidity), active resistance to instructions or attempts to be moved (catatonic negativism), the assumption of inappropriate or bizarre postures (catatonic posturing), or purposeless and unstimulated excessive motor activity (catatonic excitement)" (American Psychiatric Association, 2000). As catatonic patients are immobile because of a marked lack of reactivity to the environment, mobility aids that make facilities useful and accessible for the handicapped and frail are unlikely to be of any assistance. As it happens, design for physical accessibility is often mentioned among the requirements of mental health care projects (Goldie et al, 1989, WLHE, 1997) but a generic approach cannot suffice as a means to overcome the restrictions of movement caused by catatonia.

Accessibility issues in facilities for the mentally ill are be more complex than the mere incorporation of universal accessibility devices and, despite construction for universal accessibility standards, patients remain 'handicapped by the buildings' as Goldsmith (1976) first said in his generic comments on disability and architecture. As Peace (1998) posited, accessibility in health care environments is compromised for reasons other than physical competence. She categorised factors relating to access in a care environment according to organisation or design; time; control over space; and responsibility or accountability. If buildings fail to address the specific needs of mentally ill patients, then they might be unnecessarily restricted because of the model of care and management issues, such as restrictions on the use of external areas in the name of safety.

Over the years, limitations on the ways that mobility is restricted because of disabilities have been redressed in healthcare generally and is gradually evolving to encompass the more complex behavioural issues. In the mid 1990s, physical disability, in the sense of accessibility, became an essential concern in the design of public architecture in the UK following the implementation of Part 3 of the Disability Discrimination Act (1995). Moving a step further, Scher (1996) combined the ideas of 
access and way-finding under one argument to enhance communication in health care facilities.

The mentally ill are restricted in psychiatric settings because of the ways their conditions are understood and because of the risks that are associated with their symptoms (whether real or imagined). For example, sectioned clients in a closed ward are rarely able to leave the premises unaccompanied. This determination follows the model of care and not an architectural decision per sé. In another case, it can be that staff might not allow clients to use a courtyard that has been designed for patients to use because of the perceived risk of clients' absconding because the courtyard doesn't appear to be secure, or has not proven to be secure enough in the past or because supervisory staff are unavailable (Chrysikou, 2011). In such a case, the building was not designed with the actual safety and security levels in mind and staff have had to alter the model of care in such a way that clients loose their access the outdoors. It must be noted that outdoor access is an important therapeutic tool for mental illness (see Perkins and Wagenfield's articles in this issue) thus not only is access impaired, but therapeutic benefit is also compromised in the name of safety. Despite the welldocumented benefits of providing outdoor areas, in the UK new units might still lack direct access to open spaces (Brook, 2002). Architects with experience of secure environments have recognised garden accessibility as a major design aspect (Nightingale, 2002, Smith 2002). Additionally, the Royal College of Psychiatrists (1998) highlighted the need for secure and protected external areas for ward-bound clients. Inpatients also face problems with accessibility due to staffing regimes and models of care within day centres (Rogers et al, 1993, Chrysikou, 2011).

This paper also critically questions the adequacy of universal design aids as the main way to deal with accessibility in facilities for the adult mentally ill in the community. It is argued that the design aspects relating to accessibility have to take into consideration the patient to staff dynamics of these facilities, in order to comply with the therapeutic role of the facility.

\section{Methodology}


Topics related to restrictions of movement and the use of universal accessibility devices are extracted from post-occupancy studies of several mental healthcare units. These are discussed and analysed. Mobility restrictions imposed by the therapist or by law are excluded, except as they are the result of a poor architectural fit with the model of care.

The data is part of a broader exploratory study of facilities for mental healthcare, which used empirical, multi-disciplinary, comparative and user inclusive methods (Chrysikou, 2011), exploring the extent to which the environment itself could be considered therapeutic.

The empirical part of the research took place in France and the UK, two countries with considerable differences regarding their healthcare policies and systems, procurement processes and diagnostic patterns. This was intended to increase the generalisation value of the findings. The types of facilities chosen for the study were, for each country, the closest to acute care facilities among those providing accommodation in the community. It was important that the locus in both contexts should be in the community, as hospitals are strongly associated with the medical model and have not incorporated several aspects of the more integrated models of care that developed after the Psychiatric evolution. It was also important that the locus in each country should be the first tier of community care closest to an acute episode, as this is the stage that mentally ill people tend to be more vulnerable and need more support and therefore they might find it more difficult to cope with obstacles of their environment. In France this was the Foyers de Post Cure, and in the UK it was the ward of the Community Mental Health Centres. There were significant differences in the facilities even when they were in the same country (Marie-Cardine et al, 1992, Poggi et al 1995, Verdoux, 2007). They varied in terms of models of care and management, therapeutic functions, and building typology.

Five units in each country were studied, and the sample of people involved included 50 (non-medical) staff members and 65 patients who were willing and able to participate. Prior to conducting the fieldwork, a pilot study was carried out in a facility, which was not included in the final sample. The studies received ethics approval. 
Data was collected using semi-structured interviews with patients (rather than using doctors as mediators and advocates) and with staff. Data was also collected using architectural audits of the buildings. The latter included a descriptive approach to the facilities in relation to their respective models of care and an extensive architectural checklist. Needless to say, the research produced a considerable amount of data and discussing all of it is beyond the scope of this paper.

\section{Results}

The data identifies three areas of particular concern where freedom of mobility is hindered (in no reported cases did this include a conventional disability). The first is access to outdoor areas that were intended for patient use. The second is vertical circulation (stairs in multi-level units). And last but not least, bathrooms.

\subsection{Access to outdoor areas}

Outdoor access is often limited in mental health facilities for reasons other than physical mobility. Because limits are imposed by the models of care, which are tailored for each unit, they aren't universal. For example in France, acute patients are treated first in psychiatric hospitals, then in Foyers. In the UK, acute patients are treated in community based mental healthcare facilities even if they were sectioned by a magistrate. Thus, most acute patients in the UK will be in community based facilities, under magistrates' orders and will not be free to come and go as they please. On the other hand, in France for patients in community based facilities the opposite is true (Marie-Cardine et al, 1992, Lavaud, 1993, Lombard, 1993). Given these constraints, outdoor area accessibility is of more significance in the UK, because otherwise clients tend to be ward-bound.

Four out of ten of the case studies over both countries have no garden, and three have no outdoor space at all. Most of the time this is because they are in the inner cities. In France the lack of a garden or an external area does not deprive clients of access to the outdoors, as they are generally free to come and go as they please. French foyer clients also have access to balconies and terraces, wherever they are available. This is a contrast to the prevalent models of care in the UK, where many gardens, balconies, 
terraces and other external spaces are not deemed to be secure, so clients aren't allowed to use them. Internal courtyards are generally fully accessible to the clients if they are directly accessible from the ward. But staff in three out of five wards prevent acute clients from using gardens that are separated from the community by a fence or a wall, because of fears that patients may be able to traffic drugs or other contraband or even escape. Additionally, also in acute settings, gardens often cannot be used by clients because there are not enough staff to supervise these spaces. A similar problem also occurs when psychiatric wards are located above ground level. The vertical access becomes a barrier whenever staff must leave the ward to accompany sectioned clients.

It is clear that the safety of outdoor areas is an issue, especially for wards within larger healthcare settings. But there's little consistency in what 'secure' even means. It could mean anything from 'fully enclosed' to just 'fenced'.

From the client perspective, 28 clients (43\%) stated that they did not or could not access the outdoors. In France all clients had the option of using a garden if one was available. So, even if French clients did not use these areas, it was by their own volition rather than because of an imposition of the staff or model of care. In the UK, $29 \%$ of clients did not have access to a garden because of policy restrictions, and thus did not get to use a garden at all.

\subsection{Vertical circulation}

The second area of concern that emerged from the data regards vertical circulation (stairs, lifts etc.) even in those wards that occupy a single level if not on ground level. Here it should be mentioned that only one ward in the entire sample had no vertical circulation. The other single-storey ward was located above ground level, and depended on the ground floor centre for the dining room and therapy areas. In this instance, clients had to use the stairs to go to and from the dining room twice a day, considerably limiting their free access to that area, as well as the rest of the therapy areas that are situated there. This difficulty for clients was mostly related to the organisational separation of the ward and the day areas, rather than the vertical circulation per sé. As a result, boredom is the most reported problem in the UK facilities ( $21 \%$ of clients) and is especially prevalent in facilities where the activity 
areas are inaccessible to clients for operational reasons (Peace, 1998, Chrysikou, 2011). As Golembiewski reports (in this issue) boredom can be very detrimental to mental health.

Of the French facilities, only one had fewer than three floors. Among these, only one had accessibility provisions, so patients remained isolated in bed all day if they were injured. Furthermore, it was observed that as a result of occasionally poorly designed steps, accidents were relatively frequent. There were three foyers where bedrooms were vertically separated from common areas, and again no accessibility devices such as lifts were in place to help immobile patients negotiate vertical circulation. In the four foyers that had physical accessibility design limitations, clients with mobility problems could not access the dining rooms (3/5), the common rooms (3/5) and the therapy areas (4/5).

Units built over more than one storey always raised problems for staff, who cited safety and security concerns, especially during emergencies or when clients attempted to escape. Interestingly, accessibility deficiencies were not criticised by staff for how they compromised clients' competence. Stairs and accessibility were mentioned just three times (and only in the UK) despite the fact that facilities in France are often spread across several floors. Clients did not complain about steps, apart from in a couple of isolated cases, where the patients were physically older than most of the sample group.

\subsection{Accessibility devices in bathrooms, and bathroom layout}

Finally, the third accessibility issue concerned bathrooms. Bathrooms cannot be taken for granted in a mental health facility because they are considered dangerous for psychiatric patients - and not because they might slip and accidentally fall, but because of the opportunities bathrooms provide for suicide, self harm and interpersonal violence.

One of the observed foyers in France and all the UK case studies had made provisions for disabled clients, but in one ward the wheelchair-accessible bedroom was on the ground floor, yet the hoist for assisted bathing was in one of the upper-floor bathrooms, an inconvenience heightened by the lack of an accessible lift. The facility 
had many such issues which won't be detailed here, except to mention that confusion about universal accessibility and what that means in a psychiatric milieu is very common - and not only in the sample included in this study, suggesting that accessibility might often be an afterthought with poor follow-through into design and construction.

Once again, in general, the French facilities that were included in the study did not consider universal accessibility and there was lack of accessible bathrooms (4/5) in foyers.

When staff were asked about dangerous parts in the facilities or any post-occupancy alterations that had to be made to increase safety and security, none mentioned problems from existing universal accessibility fixtures. This was not even mentioned for areas where clients spent time unsupervised, such as bathrooms or WCs. When asked about clients' competence, the only point mentioned concerned the assistance that clients need when bathing. This was in no way limited to physically disabled patients: 42 out of 50 staff members had needed to assist patients with bathing, even though the patients were fully mobile. Thus, in a mental health facility, the primary concern for mobility in bathrooms is not the provision of universal mobility aids, but the organisation of the bathroom to allow one or two staff to physically assist clients when they bathe.

\section{Findings and conclusions}

This paper argues that accessibility in mental healthcare facilities is more complex than the mere inclusion of universal accessibility devices. The study identified three areas of primary concern: Access to outdoor areas, vertical circulation and bathroom design.

In practical terms, more secure external areas are required for the acute mentally ill because when outdoor areas are not fully enclosed, are insufficiently quarantined from areas that are accessible by the general public or not directly accessible from the ward, patients are generally not allowed to use those areas. These limitations are imposed in the name of safety and security, but have repercussions that go beyond 
accessibility and design. The lack of secure outdoor areas often means that doors to external areas are kept locked, leading to an institutional feel. Furthermore, the rules that are put in place to prevent clients from outdoor areas in acute settings stifle basic liberties, best treatment practices and good health outcomes.

The development of a facility over more than a single level does not seem to limit the indoor accessibility of acute clients, provided the universal accessibility design criteria are met and unless there is organisational separation as well, such as separation of the ward from a day centre. Staircases, for instance, do not seem to be a serious limitation for the internal circulation of clients inside the facility. This was contrary to initial expectations, except in closed environments such as wards, where there is an organisational separation - such as a level change between bedrooms and the day centre, dining room or activity area was partially of fully limited due to organisational constraints.

Having said this, multilevel design is a barrier for acute clients whenever a mental health ward is not based on the ground floor and therefore had no direct access to secure outdoor areas. But even when these issues are resolved, acute care units are not recommended above ground level because (once again) they so frequently end up compromising mobility and accessibility in the name of safety and security. Obviously the same isn't true for open facilities (such as foyers) because wherever clients are admitted on a voluntary basis, there are no particular restrictions regarding accessibility nor safety and security restrictions because patients can come and go as they please.

Universal accessibility design principles are technically as important for the design of mental health facilities as any other public buildings, but in a mental health context, these principles need to be reconsidered and expanded to reflect the realities of a functioning unit. The models of care, security and safety concerns, staffing arrangements and basic knowledge catatonia must be understood before a unit is designed and constructed. Furthermore, this understanding must be reflected holistically throughout the entire facility. Unless there's due consideration, accessibility will inevitably be compromised, often in a way that client well being, safety and health outcomes are affected. 
Once again, universal accessibility design is important for the bathrooms and shower rooms, but codes should be extended to allow for catatonic patients and to enable staff to assist clients who are otherwise still fully mobile - on a practical level, this means that baths and showers must be accessible from all sides.

Although there were valid fears that universal access devices such as handrails might provide an unacceptable ligature risks (hanging), this study showed no indication that such devices really posed a problem. No reasons to avoid them emerged from this study.

One of the findings of the study was that there are discordances between the problems that mental health facilities must address every day and architectural responses to those problems. For instance, the reality is that facilities are usually understaffed, so architectural provisions such as gardens, bathrooms and staircases may become inaccessible if they are deemed to be unsafe. The rules and locked doors that staff use to prevent clients from using areas that are not directly connected with the main body of the ward is ultimately an architectural failure. Architects must be practical, wellinformed and take the responsibility to address the need for observation or safety and security wherever risks might be present, whether they are real or imagined. Not only should areas be safe and secure, they must also feel safe and secure, because some restrictions appear to be unnecessarily put on patients because of unjustified fears.

Architects should think about accessibility in the broadest possible sense and must be considerate of the realities of the psychiatric conditions that facilities must treat, the symptoms, the staffing regimes and the models of care - if not, patients will inevitably be restricted in ways that unnecessarily limit their freedom, well being and even health outcomes.

The questions around the environments for mental health facilities are complex and are in need of much more research. Even the issues around accessibility cannot be fully addressed by one paper because in all likelihood there are other aspects of the environment that affect the accessibility of mental health facilities that this research did not address, such interpersonal communication and way-finding. 
Finally, it is important to understand that architecture should bear the responsibility to increase the beneficial potential of a facility than simply fulfil a building program. Designing a unit isn't just a matter of fitting the required number of bedrooms and other essential spaces into a compelling design within budgets and time constraints. To ignore the models of care, staffing regimes, safety and security fears and realities and patient mobility issues - either conventional or specific to psychiatric conditions, means that facilities will be built to be deficient and unsuitable. Whenever any of those issues are ignored, they are met with a string of modifications, rules and routines, which compromise both accessibility and the whole model of care. Such compromises will affect patient and staff safety, patient competency, facility efficiency as well as patient and staff wellbeing. For this reason, a shift of paradigm is necessary not only for accessibility but for mental healthcare design in general.

\section{References}

American Psychiatric Association, 2000. Diagnostic and Statistical Manual of Mental Disorders (DSM-IV-TR), Washington Dc: American Psychiatric Association, chapter 5, p. 3

Aron, E. N., 1998. The Highly Sensitive Person, New York: Broadway Books.

Bell, N., 1992. Pink Doors and Doorknockers. Stirling: Dementia Service

Development Centre.

Brook, R., 2002. The impact of a caring environment. With Design in

Mind. Presentation handout. RIBA, London, Isle of Wight Healthcare NHS Trust, June 192002.

Chrysikou, E., 2011. Architecture for the mentally ill: Analysis of therapeutic spaces for clients with severe conditions. Ph.D. Bartlett School of Graduate Studies, University College London.

Dawson, S., 1993. Ironmongery and security. AJ Focus, 7 (1), pp. 11-31. 
Disability Discrimination Act, 1995. Great Britain: Department of Health, NHS, Modernisation Agency, National Primary and Care Trust Development Team ( $\mathrm{NatPaCT})$.

Goldie, N., Pilgrim, D., and Rogers, A., 1989. Community Mental Health Centres: policy and practice. London: Good Practices in Mental Health.

Goldsmith, S., 1976. Designing for the disabled. $3^{\text {rd }}$ ed. London: RIBA Publications Limited.

Golembiewski, J. in press. Lost in Space. Facilities.

Kelly, M., 1993. Designing for People with Dementia in the Context of the Building Standards. Stirling: Dementia Services Development Centre.

Lavaud, A., 1993. Les appartements therapeutiques. Soins Psychiatrie, 150, pp.12-14.

Lombard, M., 1993. Apprendre a se reprendre en charge. Soins Psychiatrie, 150, pp.47.

Mahnke, F. H., 1996. Color, Environment and Human Response John Wiley \& Sons.

Marie-Cardine, M., and Terra, J., 1992. Le secteur psychiatrique en France: un bilan dificile. Soins Psychiatrie, 142, pp14-18.

Nightingale, M., 2002. Sevenacres - the design solution. With Design in Mind.

Presentation handout. RIBA, London, Isle of Wight Healthcare NHS Trust, June 19 2002.

Peace, S., 1998. K100 Understanding Health and Social Care, Block 2: People and Places, London: Open University Press.

Perkins, N. H.. in press. Including patients, staff and visitors in the design of the 
psychiatric milieu: Notes from the field. Facilities.

Poggi D., and Carlier, I., 1995. Voyage au Pays de CMP. Soins Psychiatrie, 172, pp.12-18

Pollack, R., 1997. Technology and design for dementia. In: Judd, Marshal and Phippen (eds.), Design for Dementia. Stirling: Dementia Services Development Centre.

Rogers, A., Pilgrine, D., and Lacey, R., 1993. Experiencing Psychiatry. London: MIND.

Royal College of Psychiatrists, 1998. Not just Bricks and Mortar, [Council Report CR62], London.

Scher, P., 1996. Patient Focused Architecture for Health Care. Manchester: Faculty of Art and Design, Manchester Metropolitan University.

Shapiro, M., Parush, S., Green, M. and Roth, D., 1997. The efficiency of the "Snoezelen" in the management of children with mental retardation who exhibit maladaptive behaviours. The British Journal of Developmental Disabilities, 43, pp. 140-155.

Shapiro, M., Roth D. and Marcus, A, 2001. The effect of lighting on the behavior of children who are developmentally disabled, DISES Journal, 4, pp.19-23

Sloan Devlin, A., 1992. Psychiatric ward renovation: staff perception and patient behaviour. Environment and Behavior, 24(1), pp. 66-84.

Smith, M., 2002. Building for mental health-stick to your principles. In: RIBA, With Design in Mind. London, 19 June 2002. London: Isle of Wight Healthcare NHS Trust 
Van der Heijden, F., Tuinier, S., Arts, N., Hoogendoorn, M., Kahn, R., and Verhoeven, W. (2005). Catatonia: Disappeared or under-diagnosed?

Psychopathology,38(1),pp. 3-8.

Verdoux, H., 2007. The current state of adult mental health care in France. Eur Arch Clin Neurosci, 257, pp.64-70.

Wagenfeld, A., Roy-Fisher, C. and Mitchell, C., in press. Collaborative Design:

Outdoor Environments for Veterans with PTSD. Facilities.

WLHE, 1997. Community Based Mental Health Facilities: safety and security by design. London: West London Health Estates (WLHE).

Zeisel, J., 2006. Inquiry by Design, London: WW Norton \& Company.

Zeisel, J., 2010. I am Still Here, London: Piactus. 